\title{
METAS DE INFLAÇÃO E VOLATILIDADE CAMBIAL: UMA ANÁLISE DA EXPERIÊNCIA INTERNACIONAL COM PAINEL-GARCH ${ }^{*}$
}

\author{
Marcos Rocha ${ }^{* *}$ \\ Marcelo Curado ${ }^{* * *}$
}

Resumo: A adoção de regimes de metas inflacionárias tem como corolário o funcionamento de um regime de câmbio flutuante. Esta conexão leva alguns analistas a concluírem que um dos custos da implantação de metas de inflação é o aumento da volatilidade do câmbio. Este trabalho segue a sugestão de Edwards (2006) de que a avaliação da volatilidade cambial dentro do regime de metas deve ser feita controlandose os efeitos do regime cambial vigente. A análise, portanto, dever ser da volatilidade condicional. Este artigo analisa a volatilidade condicional estimando um modelo de PAINEL-GARCH exponencial para 20 países que oficialmente adotaram o regime de metas. Os diferentes regimes cambiais são contemplados na explicação da volatilidade como variáveis de controle, seguindo a classificação acurada de regimes cambiais proposta por Reinhart e Rogoff (2002). Assim, os resultados contrariam muitas ideias cristalizadas por parte da literatura. No caso dos países emergentes, diferentemente do resultado para países desenvolvidos, foi encontrado que a adoção do regime de metas de inflação, Ceteris paribus, reduz a volatilidade condicional da taxa de câmbio real. Apesar de aparentemente desconcertante a princípio, o resultado segue uma lógica que é desenvolvida no artigo e que, em última instância, sugere problemas de credibilidade para o comportamento singular da volatilidade para esses países. Dentro desse contexto, é destacada a conexão entre estabilidade, fear of floating e especificidades dos países emergentes, como a dimensão do pass-trough cambial sobre os preços.

Palavras-chave: volatilidade cambial; PAINEL-GARCH; metas de inflação.

\footnotetext{
* Artigo recebido em 09/03/2009 e aprovado em 10/08/2011.

** Pesquisador do IPEA, mestre em Desenvolvimento Econômico pela UFPR e doutorando em Economia da Fundação Getúlio Vargas-SP. Contato: marcosrocha@gvmail.br.

** Doutor em Economia pela UNICAMP, professor do Departamento de Economia da UFPR e Pesquisador do Programa “Cátedras para o Desenvolvimento" do IPEA. Contato: curado@ufpr.br.
} 


\section{INFLATION TARGETING AND EXCHANGE RATE VOLATILITY: AN ANALYSIS OF INTERNATIONAL EXPERIENCE WITH PANEL-GARCH}

Abstract: The inflation targeting regimes adoption has as its corollary the operation of an exchange rate floating regime. This link makes some analysts to conclude that one of the costs of the inflation targeting implementation is the increase in the exchange rate volatility. This work follows the suggestion of Edwards (2006) that the evaluation of the exchange volatility, in a inflation targeting regime context, should be made by controlling the effects of the effective exchange rate regime; the analysis to be made is, therefore, one of the conditional volatility. This paper analyzes the conditional volatility by estimating a model of exponential PANEL-GARCH for 20 countries that officially adopted the inflation targeting. The different exchange regimes are added in the explanation of the volatility as control variables, following the acute classification of exchange regimes proposed by Reinhart and Rogoff (2002). The results that arise in this work are contrary to many already crystallized ideas in the literature. In the case of the emerging countries, differently of the result for developed countries, it was found that the IT adoption, ceteris paribus, reduces the conditional volatility of the real exchange rate. In spite of this at first seemingly disconcerting result, it follows a logic explained in the paper, which suggests credibility problems as an answer for the singular behavior of the exchange rate volatility of these countries. In this context, it is outlined the link among stability, fear of floating, and other emerging countries specificities, like the dimension of the exchange rate pass-trough on the prices.

Key-words: exchange rate volatility; PANEL-GARCH; inflation targeting.

JEL: C23; F31; F33. 


\section{INTRODUÇÃO}

Um regime de câmbio flutuante é necessário para o funcionamento eficiente do regime de metas de inflação, Inflation Targeting (IT). A razão para isso é que, em uma economia de livre mobilidade dos capitais, a política monetária independente, necessária para atingir as metas de inflação estabelecidas, não pode coexistir em um regime de câmbio fixo. Essa imposição é conhecida na literatura como "Impossibilidade da Santa Trindade"'.

A conexão entre regime de metas de inflação e taxas de câmbio flutuantes leva alguns analistas a concluírem que um dos custos da implantação do regime IT é o aumento da volatilidade cambial. Edwards (2006), entretanto, aponta um problema metodológico comum em várias análises convencionais de volatilidade sob metas de inflação: muitos trabalhos têm comparado a volatilidade da taxa de câmbio sob metas de inflação com a volatilidade de um regime fixo, ou de regimes de câmbio administrados (dirty floating). Para o autor, essa não é uma comparação adequada. A avaliação deve ser conduzida separando o regime cambial da instituição das metas de inflação.

A adoção das metas de inflação aumenta a volatilidade cambial, controlando o regime cambial vigente? Essa é a questão que deve ser investigada, segundo Edwards (2006), tendo em vista que muitas comparações têm sido feitas com a avaliação da volatilidade incondicional, ou seja, sem controlar o regime cambial vigente.

Há várias maneiras de contornar esse problema. A primeira seria analisar se a adoção de metas afeta a volatilidade de câmbio condicional em países que tiveram uma taxa de câmbio flutuante, por um período de tempo prolongado, como na Austrália e no Canadá. Outra maneira seria o controle por regime de câmbio adotando as estimações de volatilidade realizadas. Em Edwards (2006) são feitos vários exercícios econométricos nessa última linha. Este artigo propõe um caminho diferente: fazer inferências no âmbito global das experiências nos países que oficialmente adotam o

\footnotetext{
${ }^{1}$ A "Impossibilidade da Santa Trindade" se constitui um dos mais importantes resultados do modelo IS-LM para uma economia aberta. Em síntese, argumenta-se que a Autoridade Monetária (AM) é incapaz de controlar simultaneamente a taxa de câmbio e o grau de liquidez doméstico, em um contexto de mobilidade dos fluxos de capital. Ao optar pelo controle da taxa de câmbio, por exemplo, através da instituição de um regime de câmbio fixo, a AM perde a autonomia na determinação da base monetária, já que seus resultados passam a depender dos movimentos do Balanço de Pagamentos. Por exemplo, se no regime de câmbio fixo, com livre mobilidade dos fluxos de capital, a AM resolver ampliar o grau de liquidez do sistema, a taxa de juros irá cair, o que promoverá a fuga de capitais de curto prazo. Nesse contexto de déficit no Balanço de Pagamentos, a opção pela fixação da taxa de câmbio nominal obriga a AM a realizar uma operação de venda de divisas no mercado de câmbio que, em última análise, acaba promovendo o enxugamento da liquidez.
} 
regime de metas para estudar a relação entre volatilidade cambial e adoção de regimes IT. Para tanto, foi estimado um modelo GARCH exponencial de painel de dados para países selecionados. Os diferentes regimes cambiais são contemplados na explicação da volatilidade como variáveis de controle.

Dado que a maior parte dos países que adota hoje IT começaram a instituir oficialmente a implantação de metas apenas no início década de 1990 e que, além disso, muitas nações adotaram, no período, poucos regimes de câmbio. Usar a técnica de dados em painel para o maior período de tempo possível se revelou o meio mais apropriado de fazer uma boa caracterização, não só da influência dos regimes cambiais sobre a volatilidade condicional do câmbio real, mas também da adoção de IT como framework monetário. Além de melhor caracterizar as experiências, o modelo de painel lida bem com o problema de escassez de informação dos dados que surge naturalmente de uma experiência macroeconômica internacional recente como o IT.

Os resultados obtidos no exercício econométrico contrariam algumas ideias cristalizadas na literatura, contribuindo desta forma para o debate sobre os efeitos da utilização do regime de metas de inflação. Em particular, vale à pena destacar a ocorrência de resultados distintos para os grupos de países analisados. No caso dos países emergentes, diferentemente do resultado encontrado para os países desenvolvidos, a adoção do regime de metas de inflação, ceteris paribus, reduz a volatilidade condicional da taxa de câmbio real. Uma possível explicação para esse resultado é que a adoção de regimes de metas de inflação - ao ampliar a credibilidade e a transparência da política monetária - contribuiu para reduzir a volatilidade cambial nos países emergentes. Dentro desse contexto, é também discutida a conexão entre estabilidade, fear of floating e especificidades dos países emergentes, como a dimensão do pass-trough cambial sobre os preços.

$\mathrm{O}$ artigo está estruturado da seguinte forma: além desta introdução, a seção 2 seguinte desenvolve uma rápida discussão da relação metas de inflação versus volatilidade cambial. A Seção 3 detalha aspectos relacionados com a base de dados utilizada para compor o painel. A Seção 4 apresenta o modelo econométrico. A Seção 5 apresenta os resultados e traça discussões. Por fim, a Seção 6 encerra o trabalho com algumas considerações finais.

\section{METAS DE INFLAÇÃO E VOLATILIDADE CAMBIAL}

Esta seção do trabalho tem como objetivo central apresentar o debate teórico e empírico sobre a volatilidade cambial no regime de metas de inflação. Vale lembrar, a título de introdução, que a característica essencial do regime de metas de inflação é que as ações da política monetária, sobretudo a fixação da taxa de juros básica, são guiadas com o 
objetivo explícito de obtenção da taxa de inflação (ou de nível de preços) previamente determinada (Svensson, 1998; Bogdanski et al., 2000)².

Em princípio, levando-se em consideração a contribuição da literatura sobre a "impossibilidade da Santíssima Trindade", que argumenta a impossibilidade de conjugar-se, em um contexto de livre mobilidade dos fluxos de capital, o controle da política monetária e das taxas de câmbio; a instituição do regime de metas de inflação deve ser acompanhada pela adoção de regimes de flutuação das taxas de câmbio nominais e reais ${ }^{3}$. Por isso, na prática, a experiência internacional de implantação do regime de metas de inflação tem sido associada à adoção formal de regimes de taxas de câmbio flutuantes ${ }^{4}$.

No entanto, a adoção formal do regime de câmbio flutuante não necessariamente implica em efetiva ampliação das flutuações das taxas de câmbio nominais e reais. O debate acadêmico sobre o tema não é novo e muito menos consensual. Helpman (1981) argumenta que a escolha do regime de câmbio não tem implicações sobre o comportamento da taxa de câmbio real. A contraposição a esses resultados é encontrada nos chamados sticky-price models, tais como os trabalhos de Dornbusch (1976) e Mussa (1982), nos quais a rigidez de preços acaba promovendo uma maior volatilidade das taxas de câmbio nominal e real em regimes de câmbio flexível do que em regimes de administração do câmbio.

Mais recentemente, Calvo e Reinhart (2002) retomaram o debate sobre a volatilidade das taxas de câmbio num regime de câmbio flutuante. Os autores argumentam que muitos países que anunciam permitir a flutuação de suas taxas de câmbio muitas vezes não o fazem, o que ficou conhecido como "medo de flutuar" (fear of floating). O trabalho sublinha os problemas de credibilidade na explicação do fear of floating. Embora os países emergentes sejam bastante heterogêneos, eles partilham de uma característica

\footnotetext{
${ }^{2}$ Apesar de esse regime monetário apresentar como preocupação central o combate à inflação (ou a manutenção da estabilidade de preços), este não deve ser entendido como um sistema necessariamente rígido e exclusivamente focado no controle da inflação, no qual a política monetária deverá seguir regras previamente estabelecidas. Bernanke e Mishkin (1997), por exemplo, sustentam que o ele não deve ser classificado como um regime de regras rígidas de condução da política monetária, mas sim como um regime no qual há algum grau de discricionariedade para a execução de políticas, ainda que o objetivo central seja o combate à inflação.

${ }^{3}$ Para uma discussão mais detalhada sobre os requerimentos para o bom funcionamento do regime de metas de inflação, ver, entre outros, Bernanke e Mishkin (1997). Para o caso dos países emergentes, uma discussão relevante sobre o tema é encontrada em Mishkin (2000).

${ }^{4}$ A Hungria é uma exceção a essa regra. O National Bank of Hungary (NBH), na implantação do regime em agosto de 2001, sustentou que a taxa de juros, além de cumprir a função de garantir a meta de inflação, também seria a responsável pela manutenção da taxa de câmbio. Para uma discussão mais detalhada sobre esse caso, ver Jonas e Mishkin (2003).
} 
comum: a relutância em deixar suas moedas flutuarem. Essa constatação faz os autores sustentarem que há pelo menos uma causa em comum para o medo de flutuar, que é característica das economias emergentes: a falta de credibilidade nas ações da autoridade monetária, particularmente em relação à condução da política monetária. Sem credibilidade, a autoridade monetária não tem de fato autoridade.

Os problemas de credibilidade podem se manifestar de diversas maneiras, incluindo aumento da volatilidade cambial e piora nas avaliações de risco sobre a capacidade de pagamento das dívidas nacionais, além de aumento da dolarização das dividas e limitação da capacidade dos bancos centrais atuarem como efetivos emprestadores de ultima instância (lenders of last resort). Todos esses fatores alimentam o medo de grandes picos de flutuação cambial e levam a uma política implícita de suavização da flutuação do câmbio, mesmo em um contexto de regime cambial flexível.

Calvo e Reinhart (2002) distinguem outras razões para o medo de flutuar. Por exemplo, as desvalorizações em países emergentes (ou fortes depreciações) tendem a ser associadas à severas recessões. A ocorrência de defaults e as dificuldades associadas ao gerenciamento dos serviços da dívida dos países podem ser aumentadas em um contexto de maior flexibilidade, em que é permitido que a taxa de câmbio flutue. Finalmente, outro elemento destacado pelos autores é que o efeito pass-through nos países emergentes é maior do que o observado nos países desenvolvidos. Essa última observação sugere, em especial para países emergentes que adotam regimes de metas de inflação, onde a preocupação dos policy-makers concernente aos movimentos de preços é particularmente alta, a tendência de suavizar os movimentos da taxa de câmbio.

Pinto e Vieira (2007) analisam algumas experiências latino-americanas com câmbio flexível e metas de inflação ${ }^{5}$. Para o Brasil, os autores encontraram evidências de que o regime de câmbio flexível não é puro. A análise da volatilidade das reservas do país sugere que a autoridade monetária tentou, ao menos, limitar a volatilidade do câmbio no período de câmbio flexível, depois de 1999, para evitar grandes pressões inflacionárias e, possivelmente, devido aos mecanismos desencadeadores de fear of floating descritos anteriormente. O trabalho fornece também evidências de aumento da volatilidade da taxa de câmbio (não condicional ao regime cambial) para esses países com a flexibilização cambial; entretanto, são encontradas também evidências de que não houve redução significativa da volatilidade de reservas para países como Brasil e Colômbia, como era de se esperar em um contexto de passagem para um regime de livre flutuação cambial.

${ }^{5}$ Os países na amostra são o Brasil, Chile, Colômbia e México. 
De forma geral, a análise de volatilidade do trabalho de Pinto e Vieira (2007) sugere que os quatro países analisados tentam de alguma forma administrar a taxa de câmbio, seja por intermédio da taxa de juros, como acontece no Chile, Colômbia e México, seja pelo uso de reservas internacionais, como acontece no Brasil e na Colômbia, toda vez que a taxa de câmbio apresenta uma evolução de desalinhamento potencial do câmbio que possa trazer pressões inflacionárias e desequilíbrios no balanço de pagamentos.

Os estudos empíricos sobre a volatilidade das taxas de câmbio em distintos regimes cambiais têm, de uma forma geral, sugerido a existência de correlação positiva entre a adoção de regime de flutuação cambial e a ampliação da volatilidade nas taxas de câmbio. Flood e Rose (1995) e Baxter e Stockman (1988) encontram esse tipo de correlação. Trabalhos que adotam uma perspectiva em longo prazo, tais como Grilli e Kaminsky (1991) e Liang (1998) têm igualmente encontrado uma correlação positiva entre a flexibilidade do regime cambial e a volatilidade da taxa de câmbio ${ }^{6}$.

Outro aspecto importante do debate refere-se às diferenças existentes entre países e regiões no que concerne à verificação da associação entre flexibilidade do regime cambial e volatilidade da taxa. Cermenõ \& Sanin (2005) realizaram um interessante estudo empírico sobre o tema para as economias do G7 e um grupo selecionado de oito economias latino-americanas ${ }^{7}$. A conclusão do trabalho é que, para o grupo de países do G7, tem-se uma forte associação entre elevação na volatilidade da taxa de câmbio e a adoção de regimes de câmbio flexíveis. O mesmo padrão, no entanto, não é observado para os países da América Latina. Os autores sustentam que a adoção de regimes mais flexíveis está associada a menor volatilidade da taxa de câmbio quando comparada com regimes menos flexíveis.

Mais recentemente, têm-se desenvolvido trabalhos que procuram analisar o caso específico da volatilidade cambial em um regime de metas de inflação. Esses estudos tentam responder a questão central: a adoção de um regime de metas de inflação aumenta a volatilidade de câmbio? Embora, a princípio, o regime de metas deva ser combinado com a adoção de um regime de flutuação cambial e aumento do uso por parte da autoridade monetária de instrumentos de controle de inflação que incrementam a volatilidade cambial, a presença de um efeito pass-through significativo sobretudo em economias emergentes - pode desencadear, dada a preocupação nesse contexto com o controle inflacionário, um fear of floating em países que utilizam o regime de metas de inflação. Além disso, o foco da análise deveria ser na avaliação

${ }^{6}$ Grilli e Kaminsky (1991) trabalham com uma série de evidências para o período que se estende de 1885 a 1986. Liang (1988) trabalha com dados de 1880 a 1997.

${ }^{7}$ São eles: Argentina, Brasil, Chile, Colômbia, México, Venezuela, Equador e Uruguai 
da volatilidade condicional, dada as experiências com regimes cambiais, como o faz Edwards (2006), por exemplo, ao estudar a relação entre volatilidade das taxas de câmbio e regime de metas de inflação. $\mathrm{O}$ autor concluiu que a adoção do regime de metas de inflação não tem promovido acréscimo na volatilidade condicional das taxas de câmbio nominais e reais ${ }^{8}$.

\section{BASE DE DADOS}

Este trabalho utiliza tanto a base de dados de taxas de câmbio reais, construída por Reinhart e Rogoff (2002), quanto sua classificação dos regimes cambiais, para o período que vai de 1971 a 2001, contemplando os países que anunciaram oficialmente a adoção de um regime de metas de inflação como forma de conduzir sua política monetária ${ }^{9}$. Os autores desenvolveram uma reclassificação dos regimes de câmbio, tanto na taxonomia quanto nos valores das séries de taxas de câmbio. Para construir a classificação foi empregada uma base de dados extensa das taxas de câmbio determinadas no "mercado paralelo". O algoritmo de classificação mais acurado dos autores levou a uma reestruturação ampla da classificação dos regimes para diversos países e períodos.

Os autores chamam a atenção para o fato de que, na maioria das vezes em que a categorização oficial aponta alguma forma de câmbio fixo, sua nova metodologia revela que o verdadeiro regime monetário em voga é algo radicalmente diferente; muitas vezes, uma variante de flutuação. De forma análoga, o trabalho revela que quando a classificação anunciada é de regime flutuante, rotineiramente a metodologia mostra que, na realidade, trata-se de uma forma de regime de certa rigidez de facto.

A base de dados tratada pelos autores é mensal; cobre o período que vai de 1946 a 2006 para 153 países. A classificação de frequência mensal dos regimes cambiais de Reinhart e Rogoff é uma virtude crucial para a construção das variáveis que compõem os painéis desse trabalho que estuda a volatilidade condicional das taxas reais de câmbio - uma frequência menor, como a anual, por exemplo, muito provavelmente prejudicaria a consistência da estimação na análise econométrica.

\footnotetext{
${ }^{8}$ Os países da amostra são Austrália, Brasil, Canadá, Chile, Israel, Coréia e México. Os dados são referentes ao período: 1988:01 a 2005:01, exceto paro o Brasil no qual é analisado o período 1994:062005:01.

${ }^{9}$ Os países do painel aqui utilizado são: Austrália, Chile, Canadá, Israel, Grã Bretanha, Suécia, Espanha, México, República Tcheca, África do Sul, Tailândia, Hungria, Noruega, Filipinas, Turquia, República Democrática da Coréia, Brasil, Colômbia, Polônia e, finalmente, Finlândia.
} 
Quadro 1 - Categorização detalhada e categorização ampla dos regimes cambiais

\begin{tabular}{|c|c|c|}
\hline Categoria de regime cambial & $\begin{array}{l}\text { Número associado à } \\
\text { categoria detalhada }\end{array}$ & $\begin{array}{l}\text { Número associado à } \\
\text { categoria ampla }\end{array}$ \\
\hline Sem separação legal & 1 & 1 \\
\hline Peg anunciado ou currency board & 2 & 1 \\
\hline $\begin{array}{l}\text { Banda horizontal pré-anunciada que seja mais } \\
\text { estreita ou igual a }+/-2 \%\end{array}$ & 3 & 1 \\
\hline Peg de facto & 4 & 1 \\
\hline Crawling peg pré-anunciado & 5 & 2 \\
\hline $\begin{array}{l}\text { Crawling peg pré-anunciado que seja mais estrei- } \\
\text { ta ou igual a }+/-2 \%\end{array}$ & 6 & 2 \\
\hline Crawling peg de facto & 7 & 2 \\
\hline $\begin{array}{l}\text { Crawling band de facto que seja mais estreita ou } \\
\text { igual a }+/-2 \%\end{array}$ & 8 & 2 \\
\hline $\begin{array}{l}\text { Crawling band pré-anunciada que seja mais } \\
\text { ampla ou igual a }+/-2 \%\end{array}$ & 9 & 2 \\
\hline $\begin{array}{l}\text { Crawling band de facto que seja mais estreita ou } \\
\text { igual a }+/-5 \%\end{array}$ & 10 & 3 \\
\hline $\begin{array}{l}\text { Banda móvel que seja mais estreita ou igual à +/- } \\
2 \% \text { (permitindo apreciação e depreciação) }\end{array}$ & 11 & 3 \\
\hline Flutuação administrada & 12 & 3 \\
\hline Flutuação livre & 13 & 4 \\
\hline Freely falling & 14 & 5 \\
\hline
\end{tabular}

Fonte: Reinhart e Rogoff (2002).

Muitos estudos utilizam uma classificação padrão de regimes cambiais, como o Annual Report on Exchange Rate Arrangements and Exchange Restrictions, publicadas pelo Fundo Monetário Internacional (FMI ${ }^{10}$. Entretanto, uma leitura mais acurada das experiências de regimes cambiais sugere que essas classificações oficiais falham muitas vezes em descrever as práticas dos países, e que o gap entre de facto e de jure pode ser extenso. Poucos estudos prévios tentaram estender a classificação oficial do FMI de quatro padrões; alguns se basearam apenas em métodos puramente estatísticos para reclassificar os grupos de práticas cambiais.

\footnotetext{
${ }^{10} \mathrm{O}$ Fundo Monetário Internacional, reconhecendo as limitações de sua classificação inicial, revisou e renovou a classificação oficial a partir de 1997, embora não tenha reavaliado sua classificação histórica depois disso. Um problema com a classificação pré-1997 que tem recebido substancial atenção na literatura recente é a frequência de episódios em que o regime é classificado como flutuante (gerenciado ou não) quando, de fato, o país tinha um regime de câmbio de facto fixo ou crawling peg.
} 
Além disso, um problema menos visível, mas igualmente sério, está relacionado com o fato de que também as taxas de câmbio oficialmente anunciadas são muitas vezes igualmente enganadoras. Ao longo da história do pós-guerra, inúmeros países têm apresentado, em outro período, controles de capitais e sistemas de regimes de câmbio múltiplos. Quando há mercados de câmbio duais ou paralelos, um regime que é oficialmente rotulado como fixo pode facilmente ser na realidade flexível ou crawling band. Quando há mercado dual, apontam Reinhart e Rogoff (2002), não é suficiente descartar somente a classificação oficial, mas também os valores das taxas oficiais, que podem ser na realidade pouco precisos e deslocados da efetiva taxa pelas quais as transações entre moedas nacionais se dão. Assim, a utilização da base de dados dos autores, tanto quanto sua taxonomia foi crucial para a acurácia do exame das transições de regime na análise de volatilidade.

A taxonomia dos arranjos de regimes cambiais inclui 14 classificações e é apresentado no Quadro 1. Uma classificação mais geral é também construída, em que os 14 tipos de regimes são agrupados em cinco categorias mais amplas. O menos flexível dos arranjos é assinalado com os menores valores na escala, seguindo o Quadro 1 entretanto, no caso da classificação ampla, é necessário tratar freely falling ${ }^{11}$ como uma categoria separada, como sugerem os autores da classificação. Por simplicidade, e seguindo Cermeño e Sanin (2005), este artigo utiliza as cinco categorias amplas em suas estimações, e denota os regimes da seguinte forma: R1 (fixo), R2 (quase-fixo), R3 (quase flexível), R4 (livre flutuação) e R5 (freely falling). Para caracterizar a adoção oficial do regime de metas de inflação, foi criada a variável categórica IT (inflation targeting), que toma valor 1 (um) no período a partir do mês de adoção do regime, e zero caso contrário.

\section{MODELO ECONOMÉTRICO}

A análise de volatilidade de variáveis macroeconômicas é, em geral, feita com modelos da família ARCH (Auto Regressive Conditional Heteroscedasticity). Esses modelos foram implementados por Engle (1982) a partir da ideia de que a variância corrente dos resíduos pode estar relacionada com o quadrado do seu nível passado

\footnotetext{
${ }^{11}$ Freely falling é uma nova categoria cunhada por Reinhart e Rogoff (2002) em que estão agrupados aqueles casos que a taxa de inflação anual é maior do que $40 \%$ e também a ocorrências de seis meses de período após episódios de crises cambiais. Nesses casos específicos, a categoria foi criada porque esses desarranjos macroeconômicos caracterizam arranjos cambiais especiais, que devem ser distinguidos da análise de regimes feita unicamente dentro de um contexto de discussão flexibilidade/rigidez. Para maiores detalhes, consultar o trabalho dos autores.
} 
ou ao quadrado dos resíduos defasados. Mais tarde, foi desenvolvido um tipo particular de modelos ARCH, os GARCH (Generalized Auto Regressive Conditional Heteroscedasticity), que trazem como vantagem adicional permitir na análise da variância heteroscedástica os efeitos tanto de natureza autorregressiva quanto os de média móvel. Em um contexto GARCH, a variância condicional dos erros do processo estocástico consiste em um processo ARMA (Autoregressive Moving Average).

Um estudo que se proponha examinar os efeitos da adoção do regime de metas sobre a volatilidade condicional do câmbio irá, naturalmente, lidar com um problema de escassez de informação da base de dados. Não só poucos países anunciaram oficialmente a institucionalização das metas (23 países) limitando o tamanho da amostra, como a experiência de regimes IT é ainda bastante recente, reduzindo o potencial de explicação da volatilidade e reduzindo possivelmente a robustez estatística de uma variável do tipo "adoção de regime de metas" - em especial quando se estuda as experiências dos países isoladamente. A saída encontrada para contornar tal escassez dos dados no que se refere à caracterização de experiências de adoção de metas de inflação, e ao mesmo tempo solução que utiliza técnicas de modelar a variância condicional das taxas de câmbio real, foi a estimação de um tipo de modelo painel GARCH ${ }^{12}$.

Apesar do seu apelo teórico e de seu sucesso empírico, a estrutura GARCH $(p, q)$ impõe algumas restrições importantes na modelagem da volatilidade. Ela não permite que a volatilidade responda de diferentes maneiras a retornos de mesma magnitude, mas diferente sinal. ${ }^{13}$ A restrição de não negatividade implica que os incrementos aos valores absolutos dos retornos resultarão sempre em maior volatilidade. Isso elimina a possibilidade de modelar um comportamento oscilatório aleatório no processo da variância.

Nelson (1991) supera essas limitações ao introduzir o GARCH Exponencial (EGARCH), em que se propõe uma evolução dos modelos de variância condicionada, definindo-os da seguinte maneira ${ }^{14}$ :

$$
Y_{t}=\sigma_{t} \varepsilon_{t}
$$

\footnotetext{
${ }^{12}$ Muitos autores apontam que a especificação $\operatorname{GARCH}(1,1)$ satisfaz adequadamente a maioria dos fenômenos financeiros, tais como o de volatilidade cambial; em um contexto de estimação de painel, em que a especificação para cada país não é possível, essa generalização é bastante razoável. Este trabalho segue a especificação que Cermeño e Sanin (2005) utilizaram para estimar um painel semelhante.

${ }^{13}$ Essa restrição é particularmente limitadora quando se é proposto fazer, como aqui, uma análise da dinâmica de volatilidade das taxas de câmbio, que são de natureza tipicamente assimétrica quanto aos seus efeitos.

${ }^{14}$ Para uma discussão mais apurada dos modelos da família ARCH, GARCH e E-GARCH, consultar a exposição didática em Greene (2000).
} 


$$
\log \sigma_{t}^{2}=\alpha_{0}+\left(1+\sum_{i=1}^{p} \alpha_{i} L^{i}\right)+\left(1-\sum_{j=1}^{q} \beta_{j} L^{j}\right)^{-1} g\left(\varepsilon_{t-1}\right)
$$

em que $L$ é uma função de $Y$ e $\sigma$ defasadas.

Para acomodar a relação assimétrica entre retornos e volatilidade, é necessário modelar $g($.$) como uma função tanto da magnitude quanto do sinal, através de uma$ combinação linear da seguinte forma:

$$
g\left(\varepsilon_{t}\right)=\theta \varepsilon_{t}+\gamma\left[\left|\varepsilon_{t}\right|-E\left|e_{t}\right|\right]
$$

Assim, contemplando as possibilidades e exigências que o estudo se propõe, o modelo estimado foi um Painel E-GARCH $(1,1)$ para período que vai de 1971 a 2001, agrupando 20 países que declaram hoje oficialmente adotar metas de inflação como framework monetário.

\section{RESULTADOS E DISCUSSÃO}

Os resultados das estimações estão dispostos nas Tabelas 1, 2 e 3. Na Tabela 1 são apresentados os resultados para todos os países da amostra, sem segregação. Para evitar a perfeita colinearidade das variáveis categóricas (a chamada armadilha da dummy), foi excluída uma variável dummy na estimação do modelo. Seguindo Cermeño e Sanin (2005), o grupo base das dummies de regimes cambiais é o regime fixo (R1). As dummies foram construídas observando-se o mês de adoção (ou abandono) de regimes cambiais e de metas de inflação (no caso da variável IT): elas detêm valor 1 (um) quando da adoção de um regime, e zero quando do abandono (ou da não implantação no período).

É importante mencionar que as especificações contemplam defasagens usuais de um período apenas para a variável dependente (ou defasagem de 12 , no caso das amostras segregadas) ${ }^{15}$ como forma de economizar no número de parâmetros a serem estimados; os processos $\mathrm{ARCH}$, em geral, envolvem diversas iterações e, muitas vezes, dado o número alto de observações da amostra necessária para compor o painel, as experimentações possíveis com mudanças de especificações do painel são limitadas.

Para todas as especificações, em relação aos processos ARCH e GARCH, é fácil observar que os parâmetros são bastante significantes. A robustez global dos

\footnotetext{
${ }^{15} \mathrm{~A}$ escolha das defasagens da variável dependente também se deu em função das dificuldades em alcançar a convergência no método de máxima verossimilhança, comum de modelos ARCH e particularmente sensível em um contexto de painel.
} 
coeficientes aparece com bons resultados na estatística Wald, em que é rejeitada a hipótese nula. Analogamente, todos os coeficientes tiveram a hipótese nula recusada a níveis de significância de $1 \%$ ou $5 \%$.

Como o foco do presente estudo está na relação entre volatilidade condicional de taxa de cambio real e a adoção do regime de metas de inflação, as relações da taxa de câmbio real média e regimes cambiais e IT dada pela equação de média condicional não é contemplada aqui. A equação de média condicional aparece na estimação mais como técnica de modelar, de forma consistente, a variância heteroscedástica. O que interessa para o propósito deste estudo são os resultados relacionados à volatilidade da taxa de câmbio real e adoção de IT (e, eventualmente, a relação entre volatilidade cambial e regimes cambiais), dispostos pela equação de variância condicional.

A Tabela 1 do painel geral, apresentada abaixo, mostra que a adoção de regimes mais flexíveis está associada a maiores níveis de volatilidade (apontado pela redução nos sinais negativos dos coeficientes na equação de variância); R5 aparece como um regime pronunciadamente correlacionado com a maior volatilidade condicional relativa (o coeficiente é alto e positivo).

Tabela 1 - Painel E-GARCH para todos os países da amostra

\begin{tabular}{|c|c|}
\hline Equação da Média Condicional & Coeficientes \\
\hline Constante & $0.2824(0.0017)^{*}$ \\
\hline $\operatorname{AR}(1)$ & $0.9978(0.00005)^{*}$ \\
\hline $\mathrm{R} 2$ & $-0.2831(0.0017)^{*}$ \\
\hline R3 & $-0.2806(0.0017)^{*}$ \\
\hline $\mathrm{R} 4$ & $-0.2693(0.0017)^{*}$ \\
\hline R5 & $-0.2782(0.0018)^{*}$ \\
\hline IT & $0.0019(0.0006)^{*}$ \\
\hline \multicolumn{2}{|l|}{ Equação de Variância Condicional } \\
\hline E-ARCH(1) & $0.0467(0.0039)^{*}$ \\
\hline E-ARCH(a) & $0.6734(0.0056)^{*}$ \\
\hline E-GARCH(1) & $0.9885(0.0003)^{*}$ \\
\hline $\mathrm{R} 2$ & $-0.1062(0.0066)^{*}$ \\
\hline R3 & $-0.0998(0.0037)^{*}$ \\
\hline $\mathrm{R} 4$ & $-0.0578(0.0037)^{*}$ \\
\hline R5 & $0.1789(0.0033)^{*}$ \\
\hline IT & $-0.1141(0.0066)^{*}$ \\
\hline Log-Likelihood & -2690.19 \\
\hline N. de observações & 7067 \\
\hline Wald $\mathrm{Chi}^{2}(6)$ & $3.54 \mathrm{E}+08$ \\
\hline Prob $>\mathrm{Chi}^{2}$ & 0.0000 \\
\hline
\end{tabular}

Fonte: Resultados da pesquisa.

Os números entre parênteses são os desvios padrões e * ** indicam 1\% e 5\% de níveis de significância, respectivamente. 
A variável mais importante, tendo em vista os objetivos deste trabalho, é o IT. O painel amplo mostra que a adoção de regime de metas tem um impacto negativo e significante na volatilidade condicional (controlada por regime cambial). $\mathrm{O}$ resultado se alinha à hipótese de Edwards (2006) de que a adoção das metas reduz a volatilidade cambial condicional.

Mais exercícios econométricos foram tentados, fazendo-se segregações da amostra do painel em países de renda alta e em emergentes ${ }^{16}$. A Tabela 2 mostra os resultados para os países desenvolvidos ${ }^{17}$.

Tabela 2 - Painel GARCH para países de renda alta

\begin{tabular}{|c|c|}
\hline Equação da Média Condicional & Coeficientes \\
\hline Constante & $0.4266(0.0033)^{*}$ \\
\hline $\operatorname{AR}(12)$ & $0.8923(0.0002)^{*}$ \\
\hline R2 & $-0.2610(0.0043)^{*}$ \\
\hline R3 & $-0.3632(0.0033)^{*}$ \\
\hline R4 & $-0.3686(0.0034)^{\star}$ \\
\hline R5 & $-0.4046(0.0033)^{\star}$ \\
\hline \multicolumn{2}{|l|}{ Equação de Variância Condicional } \\
\hline $\mathrm{E}-\mathrm{ARCH}(1)$ & $0.052(0.0218)^{*}$ \\
\hline $\mathrm{E}-\mathrm{ARCH}(\mathrm{a})$ & $1.4009(0.0335)^{*}$ \\
\hline E-GARCH(1) & $0.9718(0.0022)^{*}$ \\
\hline R2 & $0.3793(0.0396)^{*}$ \\
\hline R3 & $0.0421(0.0279)^{*}$ \\
\hline $\mathrm{R} 4$ & $-0.0235(0.0355)^{*}$ \\
\hline R5 & $0.4041(0.0267)^{*}$ \\
\hline IT & $0.0660(0.0386)^{*}$ \\
\hline Log-Likelihood & -7452.119 \\
\hline N. de observações & 2975 \\
\hline Wald $\mathrm{Chi}^{2}(6)$ & $2.06 \mathrm{E}+07$ \\
\hline Prob $>\mathrm{Chi}^{2}$ & 0.0000 \\
\hline
\end{tabular}

Fonte: Resultados da pesquisa.

Os números entre parênteses são os desvios padrões e * ** indicam 1\% e 5\% de níveis de significância, respectivamente.

\footnotetext{
${ }^{16} \mathrm{~A}$ classificação segue a distinção entre países de renda alta de um lado, e média e baixa (emergentes) de outro, de acordo com a classificação feita pelo Banco Mundial.

${ }^{17}$ Os países da amostra contemplados nessa categoria são: Austrália, Canadá, Israel, Grã Bretanha, Suécia, Espanha, República Democrática da Coréia, Finlândia.
} 
Como pode ser observado, o cenário muda: a variável IT aparece positiva, embora o coeficiente seja tênue, próximo de zero (embora estatisticamente significante). $\mathrm{O}$ resultado sugere que, para os países da amostra de renda alta, a adoção do regime de metas de inflação teve leve (próximo de zero) impacto positivo de aumento da volatilidade condicional do câmbio real, resultado diverso daquele contemplando todos os países com IT. Portanto, uma análise somente com os países emergentes - e dados os insights já relacionados com a fear of floating e credibilidade e suas relações com volatilidade já discutidos - se faz necessária para entender os resultados da análise do painel amplo.

A Tabela 3 mostra os coeficientes estimados para a amostra composta somente por países de renda média e baixa ${ }^{18}$.

Tabela 3 - Painel GARCH para Países Emergentes

\begin{tabular}{l|c}
\hline Equação da Média Condicional & Coeficientes \\
\hline Constante & $0.21058(0.0021)^{*}$ \\
\hline AR(12) & $1.0001(0.0002)^{*}$ \\
\hline R2 & $-0.0847(0.0043)^{*}$ \\
\hline R3 & $-0.2228(0.0024)^{*}$ \\
\hline R4 & $-0.2276(0.0022)^{*}$ \\
\hline R5 & $-0.3322(0.0124)^{*}$ \\
\hline Equação de Variância Condicional & \\
\hline E-ARCH(1) & $0.0313(0.0224)$ \\
\hline E-ARCH(a) & $1.5692(0.026)^{*}$ \\
\hline E-GARCH(1) & $0.9437(0.0039)^{*}$ \\
\hline R2 & $0.0945(0.0472)^{* *}$ \\
\hline R3 & $-0.1421(0.0237)^{*}$ \\
\hline R4 & $-0.3591(0.0258)^{*}$ \\
\hline R5 & $0.3274(0.0258)^{*}$ \\
\hline IT & $-0.1271(0.0689)^{*}$ \\
\hline Log-Likelihood & -3938.494 \\
\hline N. de observações & 2231 \\
\hline Wald Chi ${ }^{2}(6)$ & $3.30 \mathrm{E}+07$ \\
\hline Prob $>$ Chi ${ }^{2}$ & 0.0000 \\
\hline Fon Resutados &
\end{tabular}

Fonte: Resultados da pesquisa.

Os números entre parênteses são os desvios padrões e *, ** indicam 1\% e 5\% de níveis de significância, respectivamente.

${ }^{18}$ Os países que compõem essa amostra são: Chile, México, República Tcheca, África do Sul, Tailândia, Hungria, Peru, Filipinas, Turquia, Brasil, Colômbia e Polônia. 
Os resultados para os países emergentes diferem daquele para os países desenvolvidos. Como no modelo para países desenvolvidos, R5 é o regime que mais determina volatilidade condicional. Entretanto, a relação entre flexibilidade cambial e volatilidade não segue a mesma lógica que foi verificada para os países desenvolvidos ${ }^{19}$. Nesse contexto, R2, a variável que descreve regimes quase flexíveis, aparece contribuindo mais para a volatilidade condicional do câmbio do que os outros regimes, a priori, mais flexíveis (exceto R5, regime de freely falling que, como no painel para os países desenvolvidos, detêm o maior coeficiente de explicação da volatilidade na história das taxas de câmbio). Os resultados sugerem que o comportamento da volatilidade de câmbio para os países emergentes difere daquela dos países desenvolvidos. Em alguma medida, esse fenômeno pode ser explicado - ainda que seu entendimento mais profundo encontre-se além dos objetivos deste trabalho - pela importância das taxas de câmbio nas economias emergentes. De acordo com Edwards (2006, p. 28): "The exchange rate is one of most important macroeconomic variables in the emerging and transition countries. It affects inflation, exports, imports and economic activity".

A experiência histórica dessas economias é farta em exemplos de como a taxa de câmbio foi, mesmo em regimes formalmente flexíveis, utilizada como instrumento de política industrial e/ou econômica. Eichengreen (2004), por exemplo, destaca a importância recente da administração das taxas de câmbio nos países emergentes da Ásia como elemento de sua estratégia de promoção do crescimento. Vale lembrar, que em sua grande maioria, tais economias utilizam regimes de câmbio flutuante:

...pegged exchange rates and resistance to pressures for revaluation as their economies and current accounts strengthen have been at the center of their development strategies... There is no question that their accumulation of reserves is a concomitant of intervention in the foreign exchange market to keep their currencies down, which is in turn a concomitant of the strategy of promoting exports as a way of stimulating growth. (Eichengreen, 2004, p. 2 e 3)

Por fim, caberia destacar que o resultado encontrado é amparado pelos argumentos de Calvo e Reinhart (2000) sobre o medo de flutuar. No que se refere especificamente ao efeito pass-through, por exemplo, os autores argumentam que este é maior nos países emergentes do que nos desenvolvidos. A preocupação com a

\footnotetext{
${ }^{19}$ Como também acontece no painel de Cermeño e Sanin (2005), cujos resultados para a América Latina relacionando volatilidade cambial de regimes cambiais se apresentaram mais controversos que os do G7. A conclusão dos autores é de que o G7 e os países da América Latina experimentaram padrões marcadamente diferentes que devem estar relacionados com as suas condições macroeconômicas particulares.
} 
inflação, sobretudo no contexto mais recente, justificaria, portanto, uma preocupação com as flutuações da taxa de câmbio, elemento que, em conjunto com as observações anteriores contribuem para entender o resultado obtido na análise dos dados do painel.

Em síntese, o padrão observado pelo estudo empírico - que regimes de câmbio mais flexíveis não se encontram relacionados com a maior volatilidade cambial nas economias periféricas - pode ser, ainda que em parte, explicado pela importância da taxa de câmbio nessas economias. Edwards (2006) e Eichengreen (2004), entre outros, sublinham tal importância tanto em termos de impactos sobre a inflação quanto da promoção do crescimento a partir de estratégias do tipo export-led growth.

Também diferente é o resultado da variável de maior interesse aqui, IT. Para os países emergentes, a variável IT resulta negativa e significante. A intuição subjacente ao resultado do coeficiente é de que, no caso dos países emergentes, a adoção do regime de metas de inflação, ceteris paribus, reduz a volatilidade condicional da taxa de câmbio real. Uma explicação para este resultado encontra-se na relação existente entre a adoção de um regime de metas de inflação e os ganhos de credibilidade obtidos pela política monetária.

De forma objetiva, a adoção de regimes de metas de inflação - ao ampliar a credibilidade e a transparência da política monetária - contribuíram para reduzir a volatilidade cambial, fenômeno especialmente importante para as economias emergentes, marcadas por décadas de comportamentos errômeos e não transparentes na condução das variáveis monetárias. Esta linha de interpretação encontra respaldo em diversas contribuições da literatura econômica sobre o tema. O ponto de partida para essa discussão encontra-se nas contribuições originais de Kydland e Prescott (1977) e Barro e Gordon (1983), como sublinha Mendonça (2004):

Desde as contribuições de Kydland e Prescott (1977) e de Barro e Gordon (1983) à literatura econômica, a análise sobre a credibilidade da política econômica tornou-se um importante objeto de estudo para os economistas. Ao longo da década de 1990 o regime de metas de inflação tornou-se um instrumento utilizado por diversos países como forma de institucionalizar o objetivo prioritário da busca da estabilidade de preços e dar maior transparência à condução da política monetária. Em outras palavras, o regime de metas de inflação representa uma estrutura que tem por finalidade eliminar os problemas relacionados à inconsistência dinâmica, e, por conseguinte, conquistar credibilidade para a autoridade monetária. (Mendonça, 2004, p. 1)

Após a implantação dos regimes de metas de inflação nos anos 1990, a difusão da literatura sobre o tema foi bastante significativa. Nesse sentido, vale a pena destacar a contribuição, entre outros, de Bernanke et al. (1998) como referência na difusão do argumento de que a adoção do regime de metas de inflação contribuiu para 
elevar a credibilidade da política monetária e, portanto, reduzir a incerteza sobre o comportamento de um amplo conjunto de variáveis macroeconômicas ${ }^{20}$.

\section{CONSIDERAÇÕES FINAIS}

A adoção de regimes de metas de inflação tem como corolário a utilização do câmbio flutuante; dada à necessidade de uma política monetária independente, focada na convergência da inflação à sua meta. Essa conexão entre regimes IT e taxas de câmbio flutuantes leva alguns analistas a concluírem que um dos custos da implantação de metas de inflação é o aumento da volatilidade do câmbio. Este trabalho segue a linha de que a avaliação da volatilidade cambial dentro do regime de metas deve ser feita controlando-se os efeitos do regime cambial vigente. Em suma, a análise, seguindo Edwards (2006), deve observar o comportamento da volatilidade condicional dos países ao regime cambial.

Este artigo analisa a volatilidade condicional fazendo inferências em nível global das experiências dos países que oficialmente adotam o regime de metas para estudar a relação entre volatilidade cambial e adoção de regimes IT. Para isso, foi construída uma estimação de um modelo de GARCH exponencial de painel de dados para países selecionados. Os diferentes regimes cambiais são contemplados na explicação da volatilidade como variáveis de controle, seguindo a classificação de regimes cambiais proposta por Reinhart e Rogoff (2002).

A questão central analisada no trabalho pode ser assim sintetizada: a adoção de um regime de metas de inflação aumenta a volatilidade condicional do câmbio? $\mathrm{O}$ resultado encontrado no trabalho empírico sugere a existência de reações distintas para os grupos de países desenvolvidos e emergentes. No caso desses últimos, as evidências sugerem que a adoção do regime de metas de inflação, ceteris paribus, reduz a volatilidade condicional da taxa de câmbio real. Uma explicação para esse resultado encontra-se na relação existente entre a adoção de um regime de metas de inflação e os ganhos de credibilidade obtidos pela política monetária. Esse resultado não foi encontrado para o grupo de países desenvolvidos. Nesse caso, a implantação do regime de metas de inflação ampliou, embora o coeficiente seja próximo de zero, a volatilidade cambial.

Finalmente, é fundamental destacar os limites deste trabalho. Como todo trabalho econométrico, os resultados obtidos devem ser interpretados à luz das variáveis

\footnotetext{
${ }^{20}$ Para uma visão crítica da literatura que associa a adoção do regime de metas de inflação com ganhos de credibilidade da política monetária, ver, entre outros, Sicsú (2002).
} 
selecionadas para análise. Esses resultados dizem respeito apenas às relações existentes entre o regime de câmbio, o regime de metas de inflação e o comportamento da volatilidade cambial. É importante lembrar que a discussão teórica e empírica sobre os determinantes da volatilidade cambial extrapola significativamente o âmbito das variáveis selecionadas ${ }^{21}$.

Em síntese, e tendo como ponto de partida os limites da abordagem utilizada, o presente trabalho fornece evidências empíricas que apontam em duas direções principais: 1) Em um plano mais geral, a adoção do regime de metas de inflação, tem impactos distintos sobre o comportamento da volatilidade cambial, quando comparamos os países desenvolvidos e as economias emergentes; 2) Em um plano mais específico, as evidências sugerem que a implantação do regime de metas de inflação contribuiu para a redução da volatilidade cambial nos países emergentes, fenômeno que pode ser entendido como o resultado do aumento da credibilidade da política monetária no regime de metas para inflação.

\section{REFERÊNCIAS}

BARRO, R. J.; GORDON, D. "Rules, discretion and reputation in a model of monetary policy”. Journal of Monetary Economics, 12, North-Holland, 101-121, 1983.

BAXTER, M.; STOCKMAN. "Business cycles and exchange rate system: some international evidence”. National Bureau of Economic Research (NBER). Working Paper, n. 2.689, 1988.

BERNANKE, B.; MISHKIN, F. "Inflation targeting; a new framework for monetary policy?" Journal of Economic Perspectives, n. 2(11), 1997.

BERNANKE, B.S.; LAUBACH, T..; MISHKIN, F.S.; POSEN, A.S. Inflation targeting: lessons from the international experience. Princeton, NJ: Princeton University Press, 1998.

BOGDANSKI, J.; TOMBINI, A.; WERLANG, S. "Implementing inflation targeting in Brazil. Banco Central do Brasil”. Working Paper. n. 1, 2000.

CALVO, G.; REINHART, C. "Fear of floating”. Quarterly Journal of Economics, v. 107, n. 2, 2002.

CERMEÑO, R.; SANIN, M. "Exchange rate arrangements and volatility of real exchange rate depreciation: panel evidence for the G7 and 8 Latin American Countries". Documento de Trabajo n. 297, CIDE, 2005.

\footnotetext{
${ }^{21}$ Uma discussão mais ampla sobre o tema deve levar em consideração outros elementos, como por exemplo, as profundas alterações ocorridas no cenário internacional, em que se destaca o ganho de importância dos países emergentes no cenário internacional. A participação destes países no produto, no comércio e na atração de capital (de curto e longo prazo) em nível global aumentou consideravelmente nos últimos anos. Tais discussões encontram-se, no entanto, muito além dos limitados objetivos deste artigo.
} 
DORNBUSCH, R. "Expectations and exchange rate dynamics". Journal of Political Economy, n. 84, p. 1.161-1.176, 1976.

EDWARDS, S. “The relationship between exchange rates and inflation targeting revisited". National Bureau of Economic Research. Working Paper n. 12.163, 2006

EICHENGREEN, B. "Global imbalances and the lessons of Bretton Woods". National Bureau of Economic Research (NBER). Working Paper n. 10.497, 2004

FLOOD, R.; ROSE, A. "Fixing exchange rates: a virtual quest for fundamentals". Journal of Monetary Economics, XXXII, p. 976-994, 1999.

GREENE, W.H. Econometric Analysis, 4 ed. Upper Saddle River: Printice Hall, 2000GRILLI, V.; KAMINSKY, G. "Nominal exchange rate regimes and real exchange rate: evidence from United States and Britain, 1885-1986”. Journal of Monetary Economics. n. 27, p. 191-212, 1991.

HELPMAN, E. "An Exploration in the theory of exchange rate regimes". Journal of Political Economy. n. 89(5), p. 865-90, 1981.

JONAS, J.; MISHKIN, F. "Inflation targeting in transition countries: experience and prospects". National Bureau of Economic Research, 2003. Working Paper n. 9.667, 2003.

KYDLAND, F.E.; PRESCOTT, E.C. "Rules rather than discretion: the inconsistency of optimal plans”. Journal of Political Economic, v. 85, n. 3, 473-492, 1977.

LIANG, H. "Real exchange rate volatility-does the nominal exchange rate regime matter?" International Monetary Fund. Working paper n. 98.147, 1998.

MENDONÇA, H. "Mensurando a credibilidade do regime de metas inflacionárias no Brasil”. Revista de Economia Politica, v. 24, n. 3 (95), julho-setembro/2004.

MISHKIN, F. “Inflation targeting in emerging market countries”. National Bureau of Economic Research (NBER). Working paper, n. 7.618, 2000.

MUSSA, M. “A Model of exchange rate dynamics”. Journal of Political Economy. n. 90, 1982.

PINTO, A.; VILELA, F. “Câmbio flexível e metas de inflação em países selecionados da América Latina: Volatilidade e Análise VAR”. Anais do X Encontro de Economia da ANPEC Região Sul, 2007.

REINHART, C.; ROGOFF, K. “The modern history of exchange rate arrangements: a reinterpretation”. NBER Working Artigo N. 8963, 2002.

SICSÚ, J. "Expectativas inflacionárias no regime de metas de inflação: uma análise preliminar do caso brasileiro". Economia Aplicada, v. 6, n. 4, 2002.

SVENSSON, L.E. “Open economy inflation targeting”. National Bureau of Economic Research. Working Artigo. n. 6.545, 1998. 